\title{
Sacroileítis en pacientes con espondiloartritis: ¿qué rol juega la ecografía?
}

\author{
Josefina Marin ${ }^{1}$, Erika Catay², Maximiliano Bravo², David Navarta ${ }^{3}$, Johana Zacariaz', Javier Rosa', Santiago Ruta ${ }^{1}$ \\ ${ }^{1}$ Sección Reumatología, Servicio Clínica Médica, Hospital Italiano de Buenos Aires. ${ }^{2}$ Reumatologia, Consultorios Médicos Moreno, Formosa. \\ ${ }^{3}$ Reumatologia, Hospital Marcial Quiroga, San Juan. \\ En nombre del Grupo de Estudio de Ultrasonido de la Sociedad Argentina de Reumatología (GESAR Ultrasonido-ECOSAR) \\ Coordinación científica: Javier Rosa ${ }^{1,}$ Santiago Ruta ${ }^{1}$ \\ ${ }^{1}$ Sección Reumatología, Servicio Clínica Médica, Hospital Italiano de Buenos Aires.
}

\section{Introducción}

El compromiso inflamatorio de las articulaciones sacroilíacas (SI), denominado sacroileítis, es una de las características distintivas de las espondiloartritis (EsA). La radiografía de las articulaciones SI ha sido tradicionalmente utilizada para el diagnóstico, clasificación y monitoreo de las EsA. La sacroileítis radiográfica forma parte de los criterios diagnósticos de espondilitis anquilosante $(\mathrm{EA})^{1}$ y debería ser la primera técnica de imagen solicitada en pacientes con lumbalgia crónica de tipo inflamatoria y sospecha de EsA. El objetivo principal radica en hallar cambios radiológicos (sacroileítis unilateral grado 3 o 4 o bilateral grado $\geq 2$ ) que permitan o no clasificar al paciente como portador de una $\mathrm{EA}^{2,3}$. De todos modos, la radiología convencional muestra las consecuencias del proceso inflamatorio a nivel de las articulaciones SI y los cambios encontrados suelen ser tardíos, lo cual puede retrasar el diagnóstico de la enfermedad en 6 a 8 años desde el inicio de los síntomas'.

Durante las últimas décadas, la resonancia magnética (RM) ha adquirido un rol protagónico en la detección de edema óseo como signo de lesión inflamatoria activa a nivel de las articulaciones SI. Esto condujo a la incorporación de la RM dentro de los criterios ASAS (Assessment of SpondyloArthritis International Society) para clasificar a los pacientes con EsA axial ${ }^{5}$.
En la actualidad, la RM puede ser considerada como método de elección para demostrar cambios inflamatorios en las articulaciones SI y es útil tanto para el diagnóstico precoz como para cuantificar la actividad de la enfermedad ${ }^{2}$. Sin embargo, muchas veces la disponibilidad de esta modalidad de imágenes es limitada, es una técnica que consume tiempo y muchas veces costosa ${ }^{6}$.

La ecografía musculoesquelética aplicada al campo de la Reumatología ha generado gran interés entre los reumatólogos durante las últimas décadas, principalmente en la evaluación del compromiso inflamatorio de diferentes artropatías. Su utilidad para valorar el compromiso inflamatorio a nivel de las articulaciones SI no está totalmente aclarada?

En el presente trabajo, realizaremos una breve descripción anatómica de las articulaciones SI y de la técnica ecográfica, y posteriormente, presentaremos un resumen sobre los puntos más salientes de la evaluación ecográfica en las articulaciones SI, acorde a la literatura disponible.

\section{Anatomía y técnica ecográfica}

Anatomía.LaarticulaciónSI esconsideradaanatómicamente como una sínfisis, es decir, una verdadera articulación diartrodial, que tiene como función principal dar soporte 
al esqueleto axial y facilitar el parto ${ }^{8}$. Está compuesta básicamente por la unión de 2 fragmentos óseos (carillas articulares) que llevan su nombre en conjunto: los huesos sacro e ilíaco. En su tercio distal se encuentra cubierta por cartílago hialino, además del característico fibrocartílago? La superficie articular es irregular, presenta numerosas crestas y leves depresiones que minimizan el movimiento y mejoran la estabilidad. Sin embargo, la estabilidad primaria se atribuye a la acción de los ligamentos adyacentes y a su reducida movilidad ${ }^{9,10,11}$.

La articulación SI en su tercio distal se encuentra separada por una pequeña cavidad sinovial a nivel dorsocaudal. Esta localización adquiere un interés especial debido a que es un sitio de inflamación frecuente en las EsA $^{11}$.

Técnica ecográfica. El paciente debe colocarse en decúbito prono en una posición relajada y sin tensión. Posteriormente, se coloca la sonda en un plano transversal, respecto al eje largo de la columna, a nivel de la apófisis espinosa de la $5^{\circ}$ vértebra lumbar (prominencia hiperecogénica). El transductor debe ser movido en sentido caudal y lateral hasta reconocer el $1^{\circ}$ agujero sacro (hendidura hipoecogénica); desde este punto, hacia fuera, se evidencia en primer lugar la cresta sacra (prominencia hiperecogénica) y a su lado una nueva hendidura hipoecogénica que corresponde a la articulación SI; más hacia lateral aún, aparece el hueso ilíaco que a ese nivel se encuentra por encima del nivel del sacro. Si continuamos bajando, se reconoce el $2^{\circ}$ agujero sacro (hendidura hipoecogénica) y hacia la parte lateral, nuevamente la articulación SI, que a este nivel encuentra al hueso ilíaco por debajo del nivel del sacro ${ }^{11}$.

La ecografía debe incluir, como en cualquier otra área articular, una evaluación en escala de grises, y luego la utilización de la técnica Doppler. La ecografía en escala de grises se puede realizar con transductores lineales o convexos, dependiendo del morfotipo del paciente, y dado que se trata de una estructura profunda, se deben utilizar frecuencias bajas a intermedias (entre 3 a $10 \mathrm{MHz}$ de frecuencia). Respecto al Doppler, se puede utilizar el Doppler color o el Doppler de poder. La señal Doppler se puede originar por la presencia de vasos fisiológicos, por ejemplo a nivel de los agujeros sacros, y puede confundir con la presencia de aumento anormal de la vascularización debido al compromiso inflamatorio a nivel de las articulaciones SI. Por tal motivo, es importante el conocimiento anatómico de dichos vasos y por otro lado conocer la medición del índice de resistencia (IR), con la utilización del Doppler espectral. Esto último nos permite discriminar la presencia de una señal proveniente de vasos con resistencias altas de aquellos pequeños vasos con baja resistencia (IR menor a 0,7) generados como consecuencia de la neoangiogénesis debida al compromiso inflamatorio. El IR es un valor numérico que representa la cantidad de flujo diastólico, el cual es directamente proporcional a la resistencia vascular periférica. Podemos inferir entonces que valores bajos de IR significan baja resistencia al flujo y nos indica la presencia de un proceso inflamatorio ${ }^{12,13,14}$.

\section{Concepto ecográfico de sacroileítis}

La presencia de sacroileítis desde el punto de vista ecográfico puede ser definida:

1) De acuerdo a la evaluación en escala de grises, como la presencia de derrame articular acorde a la definición preliminar de OMERACT (Outcomes Measures in Rheumatology): material anormal intraarticular hipoecoico o anecoico, que es desplazable y compresible, pero que no exhibe señal Doppler ${ }^{15,5}$.

2) De acuerdo a la evaluación con técnica Doppler, como la presencia de un aumento anormal de la vascularización a nivel de las articulaciones SI (Figura), la cual se define por la presencia de señal Doppler en el área de dichas articulaciones con un IR bajo (menor a 0,7) ) $^{16,17,18,19}$.

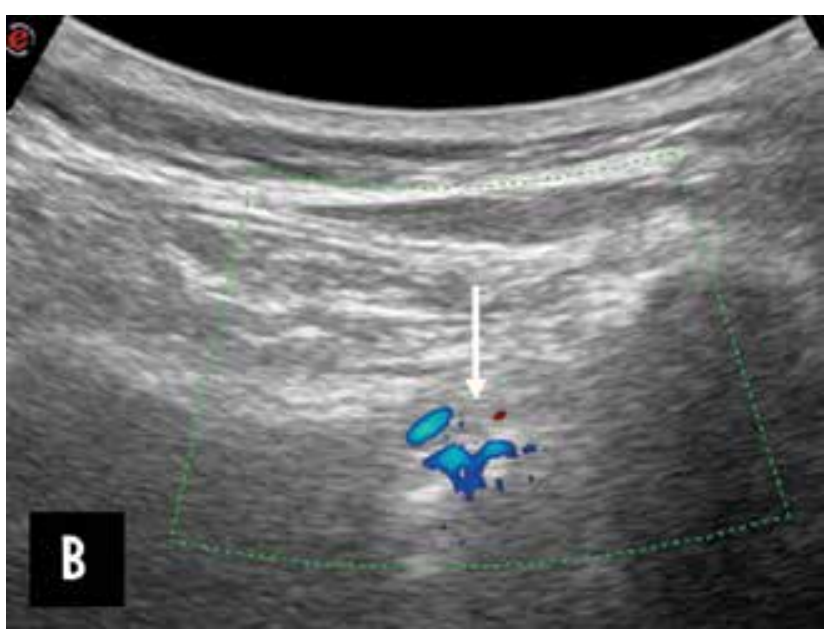

Figura. La flecha evidencia el aumento anormal de la vascularizacion por la presencia de señal Doppler a nivel de una articulación sacroilíaca en un paciente con diagnóstico de espondiloartritis axial. 


\section{Resumen de la literatura más destacada}

La evaluación ecográfica de las articulaciones SI en comparación con el examen clínico. Spadaro y cols. ${ }^{15}$ realizaron un estudio observacional de casos (45 pacientes con EsA, con y sin dolor lumbar de tipo inflamatorio) y controles (30 voluntarios sanos) comparando la presencia de derrame articular por ecografía (según OMERACT) a nivel de las articulaciones SI, con la detección clínica de sacroileítis mediante distintas maniobras semiológicas. La ecografía permitió detectar la presencia de derrame articular en el $38,9 \%$ de las articulaciones SI de pacientes con EsA, mientras que sólo fue detectado en el 1,7\% de las articulaciones SI de los controles $(p<0,001)$. Ninguna de las maniobras semiológicas, solas o combinadas, se correlacionaron con la presencia de derrame articular por ecografía. La presencia de derrame articular detectado por ecografía se correlacionó con la presencia de dolor lumbar de características inflamatorias. Este trabajo resalta la utilidad de la ecografía como un estudio de bajo costo y no invasivo, complementando al examen físico en la evaluación de las articulaciones SI.

Monitoreo terapéutico. Arslan y cols. ${ }^{17}$ compararon los hallazgos en articulaciones SI (aumento de la vascularización por Doppler e IR) de pacientes con sacroileítis activa, pacientes con artrosis y controles sanos, antes y después del tratamiento. El aumento de la vascularización alrededor de la región posterior de las articulaciones SI fue superior, y los valores de IR más bajos en los pacientes con sacroileítis activa. Los valores medios basales del IR fueron 0,62 \pm 0,13 en sacroileítis activa, $0,91 \pm 0,09$ en artrosis, y 0,97 $\pm 0,03$ en voluntarios sanos $(p<0,001$ entre los distintos grupos evaluados). Luego del tratamiento, los valores del IR cambiaron (aumentaron a una media de 0,91 $\pm 0,07$ ) en forma significativa en aquellos pacientes con sacroileítis activa.

Jiang y cols. ${ }^{16}$ estudiaron la señal Doppler de poder y el IR a nivel de las articulaciones SI de 55 pacientes con EA activa, antes y 3 meses después del tratamiento con infliximab. Encontraron cambios significativos en los dos parámetros, con disminución de la señal Doppler de poder e incremento en el IR.

Unlü y cols. ${ }^{18}$ evaluaron también el cambio en el IR antes y después de la terapia con inhibidores del factor de necrosis tumoral alfa (iFNT $\alpha$ ) en 39 pacientes con EA y encontraron, al igual que el trabajo previo, un cambio (incremento) significativo en el IR.
Propiedades diagnósticas de la ecografía con Doppler utilizando la resonancia magnética como método de referencia para la detección de sacroileitis. Klauser y cols..$^{20}$ evaluaron el valor de la ecografía Doppler con y sin contraste para la detección de sacroileítis activa en pacientes con dolor lumbar inflamatorio (n: 103), utilizando a la RM como método de referencia. Consideraron como sacroileítis ecográfica a la presencia de señal Doppler a nivel de la articulación SI, y tomando en cuenta la zona articular con mayor concentración de señal Doppler, se graduó a través de una escala subjetiva semicuantitativa, con un puntaje de 0 a 4 . La sensibilidad de la ecografía sin contraste fue del $17 \%$, con una especificidad del $96 \%$, un valor predictivo positivo (VPP) del $65 \%$ y un valor predictivo negativo (VPN) del $72 \%$. Con la administración del contraste la sensibilidad fue del $94 \%$, la especificidad del $86 \%$, el VPP del $78 \%$ y el VPN del $97 \%$. El área bajo la curva ROC de la ecografía con y sin contraste para la detección de sacroileítis activa, utilizando la RM como método de referencia, fue de 0,89 y 0,61 , respectivamente $(p<0,0001)$. Si bien este trabajo demostró que la utilización del contraste mejora la sensibilidad de la ecografía para la detección de sacroileítis activa y que además tiene un aceptable valor predictivo negativo, debemos recordar que la utilización de contraste no solo aumenta los costos, sino que también aumenta los tiempos de realización del estudio, y no está exento de eventos adversos (aunque este trabajo no mostró un incremento en el número de eventos adversos).

Mohammadi y cols. ${ }^{21}$ evaluaron las propiedades diagnósticas de la ecografía con técnica Doppler para la detección de sacroileítis activa en pacientes con EA (n: 51) y controles (n: 30), utilizando a la RM como método de referencia. La ecografía mostró una sensibilidad del $82 \%$, una especificidad del $92 \%$, un VPP del $91 \%$ y un VPN del $84 \%$ para la detección de sacroileítis activa.

Alakendu Ghosh y cols. ${ }^{19}$ evaluaron las características ecográficas a nivel de las articulaciones SI en pacientes con dolor lumbar inflamatorio sin hallazgos radiográficos (n: 29) y controles (n: 32), y lo correlacionaron con los hallazgos de la RM. Establecieron tres parámetros ecográficos para definir sacroileítis: 1) Hiperecogenicidad del espacio articular en escala de grises; 2) Aumento anormal de la vascularización por técnica Doppler (se seleccionó el área con mayor número de señales de flujo en cada articulación sacroilíaca y se cuantificaron las mismas en esa zona); 3) IR bajo de acuerdo al Doppler espectral. Tanto la presencia de un incremento en el número de señales de flujo por Doppler $(\geq 3)$ como la presencia de un IR bajo 
$(\leq 0,605)$ mostraron un adecuado acuerdo con la RM para la detección de sacroileítis, con un coeficiente kappa de 0,816 y 0,821, respectivamente. La hiperecogenicidad del espacio articular mostró un pobre acuerdo con la RM (coeficiente k 0,4), sin embargo, fue el hallazgo más específico (95\%). El parámetro ecográfico más sensible fue la presencia de un IR bajo (94,7\%). Los autores proponen que la combinación de la presencia de 3 o más señales Doppler a nivel de la articulación SI, junto con un IR bajo $(\leq 0,605)$, debería ser considerada como el criterio ecográfico con mejores propiedades diagnósticas para detectar sacroileítis.

Infiltración de articulaciones sacroiliacas guiada por ecografía. Pekkfali y cols. ${ }^{22}$ publicaron una tasa de éxito inicial del 76,7\% (46/60) en la infiltración de las articulaciones SI bajo guía ecográfica, confirmada por fluoroscopia.

En un estudio llevado a cabo en cadáveres y en pacientes con sacroileítis para evaluar distintas referencias anatómicas para la inyección de articulaciones SI guiada por ecografía, Klauser y cols. ${ }^{23}$ concluyeron que es posible utilizar distintos puntos de referencia para la infiltración, con similar tasa de éxito comparando ambas técnicas.

\section{Conclusiones}

Las articulaciones SI presentan características únicas desde un punto de vista anatómico y funcional, que dificulta frecuentemente la evaluación del compromiso inflamatorio mediante el examen clínico y los cambios radiográficos suelen ser tardíos, llevando a retrasos en el diagnóstico. Por estas razones, la RM es en la actualidad la técnica de imágenes de elección para la evaluación del compromiso inflamatorio de dichas articulaciones en pacientes con EsA.

La ecografía con técnica Doppler, como método de imagen no invasivo, carente prácticamente de contraindicaciones, de fácil acceso y bajo costo operativo, podría representar una opción ante la imposibilidad de realizar o acceder a una RM de articulaciones SI. Podría considerarse como una herramienta de extensión del examen físico, tanto para el diagnóstico de sacroileítis a través de la detección de señal Doppler intraarticular con bajos IR, como así también para un posible monitoreo terapéutico. Finalmente, otras de las posibles indicaciones de la ecografía a nivel de articulaciones SI y probablemente de gran ayuda para el reumatólogo, es su utilización para la realización de infiltraciones terapéuticas guiadas.

\section{Bibliografía}

1. van der Linden S, Valkenburg HA, Cats A. Evaluation of diagnostic criteria for ankylosing spondylitis. A proposal for modification of the New York criteria. Arthritis Rheum. 1984;27(4):361-368. http://www.ncbi.nlm.nih.gov/pubmed/6231933. Accessed December 22, 2015.

2. Mandl P, Navarro-Compán V, Terslev L, et al. EULAR recommendations for the use of imaging in the diagnosis and management of spondyloarthritis in clinical practice. Ann Rheum Dis. 2015;74(7):13271339. doi:10.1136/annrheumdis-2014-206971.

3. Rudwaleit M, van der Heijde D, Khan MA, Braun J, Sieper J. How to diagnose axial spondyloarthritis early. Ann Rheum Dis. 2004;63(5):535-543. doi:10.1136/ard.2003.011247.

4. Bandinelli F, Melchiorre D, Scazzariello F, Candelieri A, Conforti D, Matucci-Cerinic M. Clinical and radiological evaluation of sacroiliac joints compared with ultrasound examination in early spondyloarthritis. Rheumatology (Oxford). 2013;52(7):1293-1297. doi:10.1093/rheumatology/ ket105.

5. Rudwaleit M, Jurik AG, Hermann K-GA, et al. Defining active sacroiliitis on magnetic resonance imaging (MRI) for classification of axial spondyloarthritis: a consensual approach by the ASAS/OMERACT MRI group. Ann Rheum Dis. 2009;68(10):1520-1527. doi:10.1136/ard.2009.110767.

6. Guglielmi G, Scalzo G, Cascavilla A, Carotti M, Salaffi F, Grassi W. Imaging of the sacroiliac joint involvement in seronegative spondylarthropathies. Clin Rheumatol. 2009;28(9):1007-1019. doi:10.1007/ s10067-009-1192-1.

7. OstergaardM.Canimaging beused for inflammatory arthritis screening? Semin Musculoskelet Radiol. 2012;16(5):401-409. doi:10.1055/s-0032-1329893.

8. Dolor de la articulación sacroilíaca. Anatomía, Diagnóstico y Tratamiento. http://scielo.isciii.es/ pdf/dolor/v15n3/revision2.pdf. Accessed October 7, 2015.

9. Forst SL, Wheeler MT, Fortin JD, Vilensky JA. The sacroiliac joint: anatomy, physiology and clinical significance. Pain Physician. 2006;9(1):61-67. http://www.ncbi.nlm.nih.gov/pubmed/16700283. Accessed October 7, 2015. 
10. Tuite MJ. Facet joint and sacroiliac joint injection. Semin Roentgenol. 2004;39(1):37-51. http://www. ncbi.nlm.nih.gov/pubmed/14976836. Accessed October 7, 2015.

11. Sáenz-Navarro I, Möller I, Iagnocco A, Naredo E. Ultrasound assessment of the sacroiliac joint. J Clin Ultrasound. 2011;39(2):93-94. doi:10.1002/ jcu. 20773.

12. Naredo E, Monteagudo I. Doppler techniques. Clin Exp Rheumatol. 32(1 Suppl 80):S12-S19. http://www.ncbi.nlm.nih.gov/pubmed/24529255. Accessed December 27, 2015.

13. Torp-Pedersen ST, Terslev L. Settings and artefacts relevant in colour/power Doppler ultrasound in rheumatology. Ann Rheum Dis. 2008;67(2):143-149. doi:10.1136/ard.2007.078451.

14. Porta F, Radunovic G, Vlad V, et al. The role of Doppler ultrasound in rheumatic diseases. Rheumatology (Oxford). 2012;51(6):976-982. doi:10.1093/rheumatology/ker433.

15. Spadaro A, Iagnocco A, Baccano G, Ceccarelli F, Sabatini E, Valesini G. Sonographic-detected joint effusion compared with physical examination in the assessment of sacroiliac joints in spondyloarthritis. Ann Rheum Dis. 2008;68(10):1559-1563.doi:10.1136/ ard.2008.093351.

16. Jiang Y, Chen L, Zhu J, et al. Power Doppler ultrasonography in the evaluation of infliximab treatment for sacroiliitis in patients with ankylosing spondylitis. Rheumatol Int. 2013;33(8):2025-2029. doi:10.1007/s00296-013-2682-7.

17. Arslan H, Sakarya ME, Adak B, Unal O, Sayarlioglu M. Duplex and color Doppler sonographic findings in active sacroiliitis. Am J Roentgenol. 1999;173(3):677-680. doi:10.2214/ajr.173.3.10470902.

18. Unlü E, Pamuk ON, Cakir N. Color and duplex Doppler sonography to detect sacroiliitis and spinal inflammation in ankylosing spondylitis. Can this method reveal response to anti-tumor necrosis factor therapy? J Rheumatol. 2007;34(1):110-116. http://www.ncbi.nlm.nih.gov/pubmed/17216679. Accessed October 7, 2015.

19. Ghosh A, Mondal S, Sinha D, Nag A, Chakraborty S. Comment on: Ultrasonography as a useful modality for documenting sacroiliitis in radiographically negative inflammatory back pain: a comparative evaluation with MRI: reply. Rheumatology. 2015;54(8):1535-1535. doi:10.1093/rheumatology/ kev127.

20. Klauser A, Halpern EJ, Frauscher F, et al. Inflammatory low back pain: high negative predictive value of contrast-enhanced color Doppler ultrasound in the detection of inflamed sacroiliac joints. Arthritis Rheum. 2005;53(3):440-444. doi:10.1002/art.21161.

21. Mohammadi A, Ghasemi-rad M, Aghdashi M, Mladkova N, Baradaransafa P. Evaluation of disease activity in ankylosing spondylitis; diagnostic value of color Doppler ultrasonography. Skeletal Radiol. 2012;42(2):219-224. doi:10.1007/s00256-012-1412-7.

22. Pekkafahli MZ, Kiralp MZ, Baçekim CC, et al. Sacroiliac joint injections performed with sonographic guidance. J Ultrasound Med. 2003;22(6):553-559. http://www.ncbi.nlm.nih.gov/ pubmed/12795552. Accessed October 14, 2015.

23. Klauser A, DeZordo T, Feuchtner G, et al. Feasibility of ultrasound-guided sacroiliac joint injection considering sonoanatomic landmarks at two different levels in cadavers and patients. Arthritis Rheum. 2008;59(11):1618-1624. doi:10.1002/ art.24204. 